\title{
A note on quasi-bi-slant submanifolds of Sasakian manifolds
}

Received: 8 September 2020 / Accepted: 1 August 2021 / Published online: 19 August 2021 (C) The Author(s) 2021

\begin{abstract}
The object of the present paper is to study the notion of quasi-bi-slant submanifolds of almost contact metric manifolds as a generalization of slant, semi-slant, hemi-slant, bi-slant, and quasi-hemi-slant submanifolds. We study and characterize quasi-bi-slant submanifolds of Sasakian manifolds and provide nontrivial examples to signify that the structure presented in this paper is valid. Furthermore, the integrability of distributions and geometry of foliations are researched. Moreover, we characterize quasi-bi-slant submanifolds with parallel canonical structures.
\end{abstract}

Mathematics Subject Classification $53 \mathrm{C} 15 \cdot 53 \mathrm{C} 25 \cdot 53 \mathrm{~B} 20 \cdot 57 \mathrm{R} 30$

\section{Introduction}

Presently, the theory of submanifolds has gained prominence in computer design, image processing, economic modeling, as well as in mathematical physics and mechanics. These extensive applications of this topic make it an active and interesting field of research for geometers. The notion of the geometry of submanifolds begins with the idea of the extrinsic geometry of the surface and it is developed for ambient space with time.

First, the notion of slant submanifolds of almost Hermitian manifolds was studied by Chen [7] as a natural generalization of holomorphic immersions and totally real immersions. Chen collected many consequent results on slant submanifolds in his book [5]. Several examples of slant submanifolds in complex Euclidean spaces $C^{2}$ and $C^{4}$ are given by Chen and Tazawa $[8,9]$. On the other hand, using this notion Lotta introduced slant immersion of a Riemannian manifold into almost contact metric manifold in $[15,16]$. Bejancu and Papaghiuc studied the semi-invariant submanifolds of Sasakian manifold [1]. In the course of time, this interesting subject has been studied broadly by several geometers [7,11,13,14,20,22].

Cabrerizo and his co-authors [4] studied slant submanifolds of Sasakian manifold and have given several examples of such submanifolds. Semi-slant and bi-slant submanifolds of an almost contact metric manifold were studied by Cabrerizo et al. [3]. Chen et al. [22] investigated bi-slant submanifolds in Kaehler manifolds. Primarily, the hemi-slant submanifolds were known as anti-slant submanifolds. Later, Sahin [21] named these submanifolds as hemi-slant submanifolds. Hemi-slant submanifolds are one of the classes of bi-slant submanifolds. Recently, pseudo-slant submanifolds of Sasakian manifolds have been studied by Khan and Khan [12]. In $[18,19]$, we have studied and characterized quasi-hemi-slant submanifolds of Sasakian and cosymplectic manifolds and we have given several examples of such immersions.

R. Prasad · S. K. Verma $(\bowtie)$

Department of Mathematics and Astronomy, University of Lucknow, Lucknow, UP 226007, India

E-mail: skverma1208@gmail.com

R. Prasad

E-mail: rp.manpur@rediffmail.com 
Inspired from the good and interesting ideas of the above studies, we introduce the notion of quasi-bi-slant submanifolds of Sasakian manifolds as a generalization of slant, semi-slant, hemi-slant, and quasi-hemislant submanifolds, in which the tangent bundle consists of one invariant and two slant distributions and the characteristic vector field.

The present paper is organized as follows: In Sect. 2, we mention the basic definition and some properties of Sasakian manifolds. In Sect. 3, we define the notion of quasi-bi-slant submanifolds, giving a non-trivial example and obtain some basic results for the next sections. Section 4 deals with necessary and sufficient conditions for the integrability of distributions. We also investigate the geometry of foliations in this section. In the last section, we characterize quasi-bi-slant submanifolds with parallel canonical structures.

\section{Preliminaries}

We consider $\widehat{M}$ is a $(2 n+1)$-dimensional almost contact manifold [10] which carries a tensor field $\phi$ of the tangent space, $1-$ form $\eta$, and characteristic vector field $\xi$ satisfying

$$
\phi^{2}=-I+\eta \otimes \xi, \quad \eta(\xi)=1,
$$

where $I: T \widehat{M} \longrightarrow T \widehat{M}$ is the identity map. We have from definition $\phi \xi=0, \eta \circ \phi=0$ and $\operatorname{rank}(\phi)=2 n$.

Since any almost contact manifold $(\widehat{M}, \phi, \xi, \eta)$ admits a Riemannian metric $g$, such that

$$
g(\phi X, \phi Y)=g(X, Y)-\eta(X) \eta(Y),
$$

for any vector fields $X, Y \in \Gamma(T \widehat{M})$, where $\Gamma(T \widehat{M})$ represents the Lie algebra of vector fields on $\widehat{M}$. The manifold $\widehat{M}$ together with the structure $(\phi, \xi, \eta, g)$ is called an almost contact metric manifold.

From the immediate consequence of (2.2), we have

$$
\eta(X)=g(X, \xi) \text { and } g(\phi X, Y)+g(X, \phi Y)=0,
$$

for all vector fields $X, Y \in \Gamma(T \widehat{M})$.

An almost contact structure $(\phi, \xi, \eta)$ is said to be normal [2] if the almost complex structure $J$ on the product manifold $\widehat{M} \times R$ is given by

$$
J\left(U, f \frac{\mathrm{d}}{\mathrm{d} t}\right)=\left(\phi U-f \xi, \eta(U) \frac{\mathrm{d}}{\mathrm{d} t}\right),
$$

where $J^{2}=-I$ and $f$ is the differentiable function on $\widehat{M} \times R$ has no torsion, i.e., $J$ is integrable. The condition for normality in terms of $\phi, \xi$, and $\eta$ is $[\phi, \phi]+2 d \eta \otimes \xi=0$ on $\widehat{M}$, where $[\phi, \phi]$ is the Nijenhuis tensor of $\phi$. A Sasakian manifold [4] is a normal contact metric manifold and every Sasakian manifold is a $K$-contact manifold. It is easy to show that an almost contact metric manifold is a Sasakian manifold if and only if

$$
\left(\widehat{\nabla}_{X} \phi\right) Y=g(X, Y) \xi-\eta(Y) X
$$

for all vector fields $X, Y \in \Gamma(T \widehat{M})$.

Also, in a Sasakian manifold, we have

$$
\widehat{\nabla}_{X} \xi=-\phi X
$$

Let $M$ be a Riemannian manifold isometrically immersed in $\widehat{M}$ and the induced Riemannian metric on $M$ is denoted by the same symbol $g$ throughout this paper. Let $A$ and $h$ denote the shape operator and second fundamental form, respectively, of immersion of $M$ into $\widehat{M}$. The Gauss and Weingarten formulas are given by [6]

$$
\widehat{\nabla}_{X} Y=\nabla_{X} Y+h(X, Y)
$$

and

$$
\widehat{\nabla}_{X} V=-A_{V} X+\nabla_{X}^{\perp} V
$$

for any vector fields $X, Y \in \Gamma(T M)$ and $V$ on $\Gamma\left(T^{\perp} M\right)$, where $\nabla$ is the induced connection on $M$ and $\nabla^{\perp}$ represents the connection on the normal bundle $T^{\perp} M$ of $M$ and $A_{V}$ is the shape operator of $M$ with respect 
to normal vector $V \in \Gamma\left(T^{\perp} M\right)$. Moreover, $A_{V}$ and the second fundamental form $h: T M \otimes T M \longrightarrow T^{\perp} M$ of $M$ into $\widehat{M}$ are related by

$$
g(h(X, Y), V)=g\left(A_{V} X, Y\right),
$$

for any vector fields $X, Y \in \Gamma(T M)$ and $V$ on $\Gamma\left(T^{\perp} M\right)$.

The mean curvature vector is defined by

$$
H=\frac{1}{n} \operatorname{trace}(h)=\frac{1}{n} \sum_{i=1}^{n} h\left(e_{i}, e_{i}\right),
$$

where $n$ denotes the dimension of submanifold $M$ and $\left\{e_{1}, e_{2}, \ldots, e_{n}\right\}$ is the local orthonormal basis of tangent space at each point of $M$.

A submanifold $M$ of Sasakian manifold $\widehat{M}$ is said to be totally umbilical if

$$
h(X, Y)=g(X, Y) H,
$$

where $H$ is the mean curvature vector. If $h(X, Y)=0$ for all $X, Y \in \Gamma(T M)$, then $M$ is said to be totally geodesic, and if $H=0$, then $M$ is said to be a minimal submanifold.

\section{Quasi-bi-slant submanifolds of Sasakian manifolds}

In the present section of the paper, we define the notion of quasi-bi-slant submanifolds of almost contact metric manifolds, providing a non-trivial example, and obtain some related results for later use.

Definition 3.1 A quasi-bi-slant submanifold $M$ of almost contact metric manifold $\widehat{M}$ is a submanifold that admits three orthogonal complementary distributions $D, D_{1}$, and $D_{2}$, at the point $p \in M$, such that

(1) $T M$ admits the orthogonal direct decomposition

$$
T M=D \oplus D_{1} \oplus D_{2} \oplus\langle\xi\rangle,
$$

(2) The distribution $D$ is invariant, i.e., $\phi D=D$,

(3) $\phi\left(D_{1}\right) \perp D_{2}$ and $\phi\left(D_{2}\right) \perp D_{1}$,

(4) The distributions $D_{1}, D_{2}$ are slant with slant angles $\theta_{1}$ and $\theta_{2}$, respectively.

Let the dimension of distributions $D, D_{1}$ and $D_{2}$ are be $m_{1}, m_{2}$ and $m_{3}$, respectively. We easily observe the following cases:

(a) If $m_{1} \neq 0, m_{2}=0$ and $m_{3}=0$, then $M$ is an invariant submanifold.

(b) If $m_{1}=m_{2}=0$ and $\theta_{2}=\frac{\pi}{2}$, then $M$ is an anti-invariant submanifold.

(c) If $m_{1} \neq 0, m_{2}=0$ and $\theta_{2} \stackrel{2}{=}$, then $M$ is a semi-invariant submanifold.

(d) If $m_{1}=m_{2}=0$ and $0<\theta_{2}<\frac{\pi}{2}$, then $M$ is a slant submanifold with slant angles $\theta_{2}$.

(e) If $m_{1} \neq 0, m_{2} \neq 0, m_{3} \neq 0, \theta_{1}=0$ and $0<\theta_{2}<\frac{\pi}{2}$, then $M$ is a semi-slant submanifold.

(f) If $m_{1} \neq 0, m_{2} \neq 0, m_{3} \neq 0, \theta_{1}=\frac{\pi}{2}$ and $0<\theta_{2}<\frac{\pi}{2}$, then $M$ is a hemi-slant submanifold.

(g) If $m_{1} \neq 0, m_{2} \neq 0, m_{3} \neq 0$ and $0<\theta_{1}, \theta_{2}<\frac{\pi}{2}$, then $M$ is a bi-slant submanifold.

(h) If $m_{1} \neq 0, m_{2} \neq 0, m_{3} \neq 0,0<\theta_{1}<\frac{\pi}{2}$ and $\theta_{2}=\frac{\pi}{2}$, then $M$ is a quasi-hemi-slant submanifold.

(i) If $m_{1} \neq 0, m_{2} \neq 0, m_{3} \neq 0$ and $\theta_{1}, \theta_{2} \neq 0, \frac{\pi}{2}$, then $M$ is proper quasi-bi-slant submanifold.

This means that a quasi-bi-slant submanifold is a generalization of invariant, anti-invariant, semi-invariant, slant, semi-slant, hemi-slant, bi-slant, and quasi-hemi-slant submanifolds.

Remark 3.2 It is clear from Definition 3.1 that if $D \neq\{0\}, D_{1} \neq\{0\}$ and $D_{2} \neq\{0\}$, then $\operatorname{dim} D \geq 2$, $\operatorname{dim} D_{1} \geq 2$ and $\operatorname{dim} D_{2} \geq 2$. Therefore, for a proper quasi-bi-slant submanifold $M$ of an almost contact metric manifold $\widehat{M}, \operatorname{dim} M \geq 7$ and $\operatorname{dim} \widehat{M} \geq 11$.

Remark 3.3 The above definition can be generalized by taking $T M=D \oplus D_{\theta_{1}} \oplus D_{\theta_{2}} \cdots \oplus D_{\theta_{k}}$. Hence, we can define multi-slant submanifolds, quasi-multi-slant submanifolds, etc.

Remark 3.4 In this paper, we assume that $M$ is a proper quasi-bi-slant submanifold of Sasakian manifold $\widehat{M}$. 
Example 3.5 [4] Let $\mathbb{R}^{2 k+1}=\left\{\left(x_{1}, x_{2}, \ldots, x_{k}, y_{1}, y_{2}, \ldots y_{k}, z\right): x_{i}, y_{i}, z \in R, i=1,2, \ldots, k\right\}$. Consider $\mathbb{R}^{2 k+1}$ with the following structure:

$$
\begin{aligned}
& \phi\left(\sum_{i=1}^{k}\left(X^{i} \frac{\partial}{\partial x_{i}}+Y^{i} \frac{\partial}{\partial y_{i}}\right)+Z \frac{\partial}{\partial z}\right)=\sum_{i=1}^{k} Y^{i} \frac{\partial}{\partial x_{i}}-\sum_{i=1}^{k} X^{i} \frac{\partial}{\partial y_{i}}+\sum_{i=1}^{k} Y^{i} y_{i} \frac{\partial}{\partial z} \\
& g=(\eta \otimes \eta)+\frac{1}{4} \sum_{i=1}^{k}\left(d x_{i} \otimes d x_{i}+d y_{i} \otimes d y_{i}\right), \\
& \eta=\frac{1}{2}\left(d z-\sum_{i=1}^{k} y_{i} d x_{i}\right), \quad \xi=2 \frac{\partial}{\partial z}
\end{aligned}
$$

Then, $\left(\mathbb{R}^{2 k+1}, \phi, \xi, \eta, g\right)$ is a Sasakian manifold. The vector fields $e_{i}=2 \frac{\partial}{\partial y_{i}}, e_{k+i}=2\left(\frac{\partial}{\partial x_{i}}+y_{i} \frac{\partial}{\partial z}\right)$ and $\xi$ form a $\phi$-basis for the almost contact metric structure.

Now, we give an example of proper quasi-bi-slant submanifold in $\mathbb{R}^{13}$.

Example 3.6 Consider a 13-dimensional differentiable manifold

$$
\bar{M}=\left\{\left(x_{1}, x_{2}, \ldots, x_{6}, y_{1}, y_{2}, \ldots, y_{6}, z\right): x_{i}, y_{i}, z \in \mathbb{R} ; i=1,2, \ldots, 6\right\},
$$

with structure $(\phi, \xi, \eta, g)$ as defined in Example 3.5. Then, $(\bar{M}, \phi, \xi, \eta, g)$ become a Sasakian manifold of dimension 13. We choose the vector fields

$$
e_{i}=2 \frac{\partial}{\partial y_{i}}, e_{6+i}=2\left(\frac{\partial}{\partial x_{i}}+y_{i} \frac{\partial}{\partial z}\right), e_{13}=\xi=2 \frac{\partial}{\partial z}, \quad \text { for } i=1,2, \ldots, 6
$$

Then, $\left\{e_{1}, e_{2}, \ldots, e_{13}\right\}$ forms an orthonormal basis at each point of tangent space of $\bar{M}$.

Now, we consider a submanifold $M$ of $\bar{M}$ defined by immersion $f$ as follows:

$$
f(u, v, w, r, s, t, q)=\left(u, w, 0, s, 0,0, v, r \cos \theta_{1}, r \sin \theta_{1}, t \cos \theta_{2}, 0, t \sin \theta_{2}, q\right),
$$

where $\theta_{1}$ and $\theta_{2}$ are constant.

If we take

$$
\begin{aligned}
& Z_{1}=e_{1}, \quad Z_{2}=e_{7}, \quad Z_{3}=e_{2}, \\
& Z_{4}=\cos \theta_{1} e_{8}+\sin \theta_{1} e_{9}, \quad Z_{5}=e_{4}, \\
& Z_{6}=\cos \theta_{2} e_{10}+\sin \theta_{2} e_{12}, \quad Z_{7}=e_{13} .
\end{aligned}
$$

Then, the set $\left\{Z_{1}, Z_{2}, Z_{3}, Z_{4}, Z_{5}, Z_{6}, Z_{7}\right\}$ restricted over $M$ spans the tangent bundle $T M$ of $M$.

Obviously, we get

$$
\begin{aligned}
& \phi Z_{1}=e_{7}, \quad \phi Z_{2}=-e_{1}, \quad \phi Z_{3}=e_{8}, \\
& \phi Z_{4}=-\left(\cos \theta_{1} e_{2}+\sin \theta_{1} e_{3}\right), \quad \phi Z_{5}=e_{10}, \\
& \phi Z_{6}=-\left(\cos \theta_{2} e_{4}+\sin \theta_{2} e_{6}\right), \quad \phi Z_{7}=0 .
\end{aligned}
$$

Now, let us put $D=\operatorname{Span}\left\{Z_{1}, Z_{2}\right\}, D_{1}=\operatorname{Span}\left\{Z_{3}, Z_{4}\right\}, D_{2}=\operatorname{Span}\left\{Z_{5}, Z_{6}\right\}$.

Then, one can easily check that the distribution $D$ is invariant and the distributions $D_{1}, D_{2}$ are slant with slant angles $\theta_{1}$ and $\theta_{2}$, respectively. Also, we observe that $D, D_{1}$ and $D_{2}$, satisfy the definition of quasi-bi-slant submanifold of Sasakian manifold. Hence, submanifold $M$ defined by $f$ is a proper quasi-bi-slant submanifold of $\bar{M}$ with $\theta_{1}, \theta_{2}$ as its bi-slant angles.

Let $M$ be a quasi-bi-slant submanifold of a Sasakian manifold $\widehat{M}$. We denote the projections of $X \in \Gamma(T M)$ on the distributions $D, D_{1}$ and $D_{2}$ by $P, Q$, and $R$, respectively. Then, for any $X \in \Gamma(T M)$, we can write

$$
X=P X+Q X+R X+\eta(X) \xi .
$$

Now, put

$$
\phi X=T X+N X
$$


where $T X$ and $N X$ are tangential and normal components of $\phi X$ on $M$.

Making use of (3.1) and (3.2), we obtain

$$
\phi X=T P X+N P X+T Q X+N Q X+T R X+N R X .
$$

Since $\phi D=D$, we have $N P X=0$. Therefore, we get

$$
\phi X=T P X+T Q X+T R X+N Q X+N R X .
$$

Thus, for any $X \in \Gamma(T M)$, it is easy to see that

$$
T X=T P X+T Q X+T R X
$$

and

$$
N X=N Q X+N R X .
$$

Thus, from (3.4), we have the following decomposition:

$$
\phi(T M)=D \oplus T D_{1} \oplus T D_{2} \oplus N D_{1} \oplus N D_{2},
$$

where ' $\oplus$ ' denotes orthogonal direct sum.

Since $N D_{1} \subset \Gamma\left(T^{\perp} M\right)$ and $N D_{2} \subset \Gamma\left(T^{\perp} M\right)$, we have

$$
T^{\perp} M=N D_{1} \oplus N D_{2} \oplus \mu,
$$

where $\mu$ is the orthogonal complement of $N D_{1} \oplus N D_{2}$ in $\Gamma\left(T^{\perp} M\right)$ and it is invariant with respect to $\phi$. For any non-zero vector field $V \in \Gamma\left(T^{\perp} M\right)$, we have

$$
\phi V=t V+n V,
$$

where $t V \in \Gamma(T M)$ and $n V \in \Gamma\left(T^{\perp} M\right)$.

Lemma 3.7 Let $M$ be a quasi-bi-slant submanifold of Sasakian manifold $\widehat{M}$. Then, we obtain

$$
T D=D, T D_{1} \subset D_{1}, T D_{2} \subset D_{2}, t N D_{1} \subset D_{1}, t N D_{2} \subset D_{2} .
$$

Lemma 3.8 Let $M$ be a submanifold of Sasakian manifold $\widehat{M}$. Then, for any $X, Y \in \Gamma(T M)$ and $V \in$ $\Gamma\left(T^{\perp} M\right)$, we have

$$
\begin{aligned}
& \nabla_{X} T Y-A_{N Y} X-T \nabla_{X} Y-t h(X, Y)=g(X, Y) \xi-\eta(Y) X \\
& h(X, T Y)+\nabla_{X}^{\perp} N Y-N\left(\nabla_{X} Y\right)-n h(X, Y)=0, \\
& \nabla_{X} t V-t \nabla_{X}^{\perp} V=A_{n V} X-T A_{V} X
\end{aligned}
$$

and

$$
\nabla_{X}^{\perp} n V-n \nabla_{X}^{\perp} V=-h(X, t V)-N A_{V} X .
$$

Proof Since $\widehat{M}$ is a Sasakian manifold, we have

$$
\widehat{\nabla}_{X} \phi Y-\phi \widehat{\nabla}_{X} Y=g(X, Y) \xi-\eta(Y) X .
$$

By making use of Eqs. (2.5), (2.6), (3.2), and (3.7), we obtain

$$
\begin{aligned}
& \nabla_{X} T Y-A_{N Y} X-T \nabla_{X} Y-\operatorname{th}(X, Y) \\
& \quad+h(X, T Y)+\nabla_{X}^{\perp} N Y-N\left(\nabla_{X} Y\right)-n h(X, Y)=g(X, Y) \xi-\eta(Y) X .
\end{aligned}
$$

On comparing tangential and normal components, we have the first two results. In a similar way, we have the last two.

Taking into account Eqs. (3.2) and (3.7), and using the fact that $\phi^{2}=-I+\eta \otimes \xi$, we have the followings:

Lemma 3.9 Let $M$ be a quasi-bi-slant submanifold of a Sasakian manifold $\widehat{M}$. Then, the endomorphisms $T$ and $N$ (resp., $t$ and $n$ ) in the tangent bundle (resp., in the normal bundle) of $M$, satisfy the following identities: 
(i) $T^{2}+t N=-I+\eta \otimes \xi$ on $T M$,

(ii) $N T+n N=0$ on $T M$,

(iii) $N t+n^{2}=-I$ on $\left(T^{\perp} M\right)$,

(iv) $T t+t n=0$ on $\left(T^{\perp} M\right)$,

where I is the identity operator.

Lemma 3.10 Let $M$ be a quasi-bi-slant submanifold of a Sasakian manifold $\widehat{M}$. Then

(1) $T^{2} X=-\left(\cos ^{2} \theta_{i}\right) X$,

(2) $g(T X, T Y)=\left(\cos ^{2} \theta_{i}\right) g(X, Y)$,

(3) $g(N X, N Y)=\left(\sin ^{2} \theta_{i}\right) g(X, Y)$,

for any $X, Y \in D_{i}$, for $i=1,2$.

Proof The proof follows using similar steps as in Proposition 2.8 of [17].

Lemma 3.11 Let $M$ be a quasi-bi-slant submanifold of a Sasakian manifold $\widehat{M}$. Then

$$
h(X, \xi)=-N X \text { and } \nabla_{X} \xi=-T X \text { for all } X \in \Gamma(T M) .
$$

Lemma 3.12 Let $M$ be a quasi-bi-slant submanifold of a Sasakian manifold $\widehat{M}$. Then

(i) $g\left(\widehat{\nabla}_{X} Y, \xi\right)=g(T X, Y)$;

(ii) $g([X, Y], \xi)=2 g(T X, Y)$

for all $X, Y \in \Gamma\left(D \oplus D_{1} \oplus D_{2}\right)$.

Proof Let $X, Y \in \Gamma\left(D \oplus D_{1} \oplus D_{2}\right)$, and consider

$$
\widehat{\nabla}_{X}\{g(Y, \xi)\}=g\left(\widehat{\nabla}_{X} Y, \xi\right)+g\left(Y, \widehat{\nabla}_{X} \xi\right) .
$$

Since $Y$ is orthogonal to $\xi$, therefore from above equation, we have

$$
g\left(\widehat{\nabla}_{X} Y, \xi\right)=-g\left(Y, \widehat{\nabla}_{X} \xi\right) .
$$

Using $\widehat{M}$ is a Sasakian manifold and above equation, we have

$$
g\left(\widehat{\nabla}_{X} Y, \xi\right)=g(T X, Y) .
$$

Now

$$
g([X, Y], \xi)=g\left(\widehat{\nabla}_{X} Y, \xi\right)-g\left(\widehat{\nabla}_{Y} X, \xi\right) .
$$

Using (3.9) and (3.10), we have

$$
g([X, Y], \xi)=2 g(T X, Y) \text { for all } X, Y \in \Gamma\left(D \oplus D_{1} \oplus D_{2}\right) .
$$

This completes the proof.

\section{Integrability and totally geodesic foliations}

In this section, we examine the integrability conditions for invariant distribution $D$, and slant distributions $D_{1}$ and $D_{2}$.

Proposition 4.1 Let $M$ be a proper quasi-bi-slant submanifold of a Sasakian manifold $\widehat{M}$. Then, the invariant distribution $D$ and slant distribution $D_{i} ;(i=1,2)$ are not integrable.

Proof The proof follows using Lemma 3.12(ii).

Theorem 4.2 Let $M$ be a proper quasi-bi-slant submanifold of a Sasakian manifold $\widehat{M}$. Then, the distribution $D \oplus\langle\xi\rangle$ is integrable if and only if

$$
g\left(\nabla_{X} T Y-\nabla_{Y} T X, T Q Z+T R Z\right)=g(h(Y, T X)-h(X, T Y), N Q Z+N R Z),
$$

for any $X, Y \in \Gamma(D \oplus\langle\xi\rangle)$ and $Z \in \Gamma\left(D_{1} \oplus D_{2}\right)$. 
Proof The distribution $D \oplus\langle\xi\rangle$ is integrable on $M$ if and only if $g([X, Y], Z)=0$ for any $X, Y \in \Gamma(D \oplus\langle\xi\rangle)$ and $Z \in \Gamma\left(D_{1} \oplus D_{2}\right)$.

Now, for any $X, Y \in \Gamma(D \oplus\langle\xi\rangle)$ and $Z=Q Z+R Z \in \Gamma\left(D_{1} \oplus D_{2}\right)$, using (2.2), we have

$$
g([X, Y], Z)=g\left(\phi \widehat{\nabla}_{X} Y, \phi Z\right)+\eta\left(\widehat{\nabla}_{X} Y\right) \eta(Z)-g\left(\phi \widehat{\nabla}_{Y} X, \phi Z\right)-\eta\left(\widehat{\nabla}_{Y} X\right) \eta(Z),
$$

Using (2.4), (3.2), and $N Y=0$ for any $Y \in(D \oplus\langle\xi\rangle)$, we obtain

$$
\begin{aligned}
g([X, Y], Z) & =g\left(\widehat{\nabla}_{X} \phi Y, \phi Z\right)-g\left(\widehat{\nabla}_{Y} \phi X, \phi Z\right), \\
& =g\left(\widehat{\nabla}_{X} T Y, \phi Z\right)-g\left(\widehat{\nabla}_{Y} T X, \phi Z\right) .
\end{aligned}
$$

By making use of (2.5) and (3.4) in the above equation, we get

$$
\begin{aligned}
g([X, Y], Z)= & g\left(\nabla_{X} T Y-\nabla_{Y} T X, T Q Z+T R Z\right) \\
& +g(h(X, T Y)-h(Y, T X), N Q Z+N R Z) .
\end{aligned}
$$

This completes the proof.

Theorem 4.3 Let $M$ be a proper quasi-bi-slant submanifold of a Sasakian manifold $\widehat{M}$. Then, the distribution $D_{1} \oplus<\xi>$ is integrable if and only if

$$
\begin{aligned}
g\left(A_{N W} Z-A_{N Z} W, T X\right)= & g\left(A_{N T W} Z-A_{N T Z} W, X\right) \\
& +g\left(\nabla_{Z}^{\perp} N W-\nabla_{W}^{\perp} N Z, N R X\right)
\end{aligned}
$$

for any $Z, W \in \Gamma\left(D_{1} \oplus\langle\xi\rangle\right)$ and $X \in \Gamma\left(D \oplus D_{2}\right)$.

Proof The distribution $D_{1} \oplus\langle\xi\rangle$ is integrable on $M$ if and only if $g([Z, W], X)=0$, for any $Z, W \in$ $\Gamma\left(D_{1} \oplus\langle\xi\rangle\right)$ and $X \in \Gamma\left(D \oplus D_{2}\right)$.

Now, for any $Z, W \in \Gamma\left(D_{1} \oplus\langle\xi\rangle\right)$ and $X=P X+R X \in \Gamma\left(D \oplus D_{2}\right)$, using (2.2), we have

$$
g([Z, W], X)=g\left(\phi \widehat{\nabla}_{Z} W, \phi X\right)+\eta\left(\widehat{\nabla}_{Z} W\right) \eta(X)-g\left(\phi \widehat{\nabla}_{W} Z, \phi X\right)-\eta\left(\widehat{\nabla}_{W} Z\right) \eta(X) .
$$

Using (2.2), (2.4), 2.5, and (3.2), we obtain

$$
\begin{aligned}
g([Z, W], X)= & g\left(\widehat{\nabla}_{Z} T W, \phi X\right)+g\left(\widehat{\nabla}_{Z} N W, \phi X\right) \\
& -g\left(\widehat{\nabla}_{W} T Z, \phi X\right)-g\left(\widehat{\nabla}_{W} N Z, \phi X\right) . \\
= & -g\left(\widehat{\nabla}_{Z} \phi T W, X\right)+g\left(\widehat{\nabla}_{Z} N W, \phi X\right) \\
& +g\left(\widehat{\nabla}_{W} \phi T Z, X\right)-g\left(\widehat{\nabla}_{W} N Z, \phi X\right) .
\end{aligned}
$$

Then, from (2.6), (3.2) and Lemma 3.10, we have

$$
\begin{aligned}
g([Z, W], X)= & \cos ^{2} \theta_{1} g([Z, W], X)-g\left(A_{N W} Z-A_{N Z} W, \phi X\right) \\
& +g\left(A_{N T W} Z-A_{N T Z} W, X\right)+g\left(\nabla_{Z}^{\perp} N W-\nabla_{W}^{\perp} N Z, \phi X\right),
\end{aligned}
$$

which leads to

$$
\begin{aligned}
\sin ^{2} \theta_{1} g([Z, W], X)= & g\left(A_{N T W} Z-A_{N T Z} W, X\right)+g\left(\nabla_{Z}^{\perp} N W-\nabla_{W}^{\perp} N Z, N R X\right) \\
& -g\left(A_{N W} Z-A_{N Z} W, T X\right) .
\end{aligned}
$$

This completes the proof.

In a similar way, we obtain the following case for the distribution $D_{2} \oplus\langle\xi\rangle$ :

Theorem 4.4 Let $M$ be a quasi-bi-slant submanifold of a Sasakian manifold $\widehat{M}$. Then, the slant distribution $D_{2} \oplus\langle\xi\rangle$ is integrable if and only if

$$
\begin{aligned}
g\left(A_{N W} Z-A_{N Z} W, T X\right)= & g\left(A_{N T W} Z-A_{N T Z} W, X\right) \\
& +g\left(\nabla_{Z}^{\perp} N W-\nabla_{W}^{\perp} N Z, N Q X\right)
\end{aligned}
$$

for any $Z, W \in \Gamma\left(D_{2} \oplus\langle\xi\rangle\right)$ and $X \in \Gamma\left(D \oplus D_{1}\right)$. 
From Theorems 4.3 and 4.4, we have the following sufficient conditions for the distribution $D_{i} \oplus\langle\xi\rangle$; $(i=1,2)$ to be integrable.

Theorem 4.5 Let $M$ be a proper quasi-bi-slant submanifold of a Sasakian manifold $\widehat{M}$. If

$$
\begin{gathered}
\nabla_{Z}^{\perp} N W-\nabla_{W}^{\perp} N Z \in N D_{i} \oplus \mu, \\
A_{N T W} Z-A_{N T Z} W \in D_{i} \text { and } \\
A_{N W} Z-A_{N Z} W \in D_{i}
\end{gathered}
$$

for any $Z, W \in \Gamma\left(D_{i} \oplus\langle\xi\rangle\right)$, then the slant distribution $D_{i} \oplus\langle\xi\rangle$ is integrable, for $i=1,2$.

We now investigate the geometry of leaves of invariant and slant distributions.

Proposition 4.6 Let $M$ be a proper quasi-bi-slant submanifold of a Sasakian manifold $\widehat{M}$. Then, the invariant distribution $D$ and slant distribution $D_{i} ;(i=1,2)$ do not define a totally geodesic foliation on $M$.

Proof The proof directly follows from Lemma 3.12(i).

Theorem 4.7 Let $M$ be a proper quasi-bi-slant submanifold of a Sasakian manifold $\widehat{M}$. Then, the invariant distribution $D \oplus\langle\xi\rangle$ defines a totally geodesic foliation on $M$ if and only if

$$
\begin{aligned}
g\left(\nabla_{X} T Y, T Q Z+T R Z\right) & =-g(h(X, T Y), N Q Z+N R Z) \text { and } \\
g\left(\nabla_{X} T Y, t U\right) & =-g(h(X, T Y), n U)
\end{aligned}
$$

for any $X, Y \in \Gamma(D \oplus\langle\xi\rangle), Z \in \Gamma\left(D_{1} \oplus D_{2}\right)$ and $U \in \Gamma\left(T^{\perp} M\right)$.

Proof The distribution $D \oplus\langle\xi\rangle$ defines a totally geodesic foliation on $M$ if and only if $g\left(\widehat{\nabla}_{X} Y, Z\right)=0$ and $g\left(\widehat{\nabla}_{X} Y, U\right)=0$ for any $X, Y \in \Gamma(D \oplus\langle\xi\rangle), Z \in \Gamma\left(D_{1} \oplus D_{2}\right)$, and $U \in \Gamma\left(T^{\perp} M\right)$.

Now, for any $X, Y \in \Gamma(D \oplus\langle\xi\rangle), Z=Q Z+R Z \in \Gamma\left(D_{1} \oplus D_{2}\right)$ and using (2.2), (2.5), (3.2), and $N Y=0$, we have

$$
\begin{aligned}
g\left(\widehat{\nabla}_{X} Y, Z\right) & =g\left(\widehat{\nabla}_{X} T Y, \phi Z\right), \\
& =g\left(\nabla_{X} T Y, T Q Z+T R Z\right)+g(h(X, T Y), N Q Z+N R Z) .
\end{aligned}
$$

which gives first part of (4.5).

Now, for any $U \in \Gamma\left(T^{\perp} M\right)$ and $X, Y \in \Gamma(D \oplus\langle\xi\rangle)$, we have

$$
\begin{aligned}
g\left(\widehat{\nabla}_{X} Y, U\right) & =g\left(\widehat{\nabla}_{X} T Y, \phi U\right) \\
& =g\left(\nabla_{X} T Y, t U\right)+g(h(X, T Y), n U),
\end{aligned}
$$

which gives second part of (4.5).

Theorem 4.8 Let $M$ be a proper quasi-bi-slant submanifold of a Sasakian manifold $\widehat{M}$. Then, the slant distribution $D_{1} \oplus\langle\xi\rangle$ defines a totally geodesic foliation on $M$ if and only if

$$
\begin{aligned}
g\left(\nabla_{X}^{\perp} N Y, N R Z\right) & =g\left(A_{N Y} X, T P Z+T R Z\right)-g\left(A_{N T Y} X, Z\right) \text { and } \\
g\left(A_{N Y} X, t V\right) & =g\left(\nabla_{X}^{\perp} N Y, n V\right)-g\left(\nabla_{X}^{\perp} N T Y, V\right)
\end{aligned}
$$

for any $X, Y \in \Gamma\left(D_{1} \oplus\langle\xi\rangle\right), Z \in \Gamma\left(D \oplus D_{2}\right)$ and $V \in \Gamma\left(T^{\perp} M\right)$.

Proof For any $X, Y \in \Gamma\left(D_{1} \oplus\langle\xi\rangle\right), Z=P Z+R Z \in \Gamma\left(D \oplus D_{2}\right)$ and using (2.2), (2.4), and (3.2), we have

$$
\begin{aligned}
g\left(\widehat{\nabla}_{X} Y, Z\right) & =g\left(\widehat{\nabla}_{X} \phi Y, \phi Z\right)=g\left(\widehat{\nabla}_{X} T Y, \phi Z\right)+g\left(\widehat{\nabla}_{X} N Y, \phi Z\right) \\
& =-g\left(\widehat{\nabla}_{X} T^{2} Y, Z\right)-g\left(\widehat{\nabla}_{X} N T Y, Z\right)+g\left(\widehat{\nabla}_{X} N Y, \phi Z\right) .
\end{aligned}
$$

Then, using (2.6), (3.2), and Lemma 3.10, and the fact that $N P Z=0$, we have

$$
\begin{aligned}
g\left(\widehat{\nabla}_{X} Y, Z\right)= & \cos ^{2} \theta_{1} g\left(\widehat{\nabla}_{X} Y, Z\right)+g\left(A_{N T Y} X, Z\right) \\
& -g\left(A_{N Y} X, T P Z+T R Z\right)+g\left(\nabla_{X}^{\perp} N Y, N R Z\right), \\
\sin ^{2} \theta_{1} g\left(\widehat{\nabla}_{X} Y, Z\right)= & g\left(A_{N T Y} X, Z\right)-g\left(A_{N Y} X, T P Z+T R Z\right) \\
& +g\left(\nabla_{X}^{\perp} N Y, N R Z\right) .
\end{aligned}
$$


Similarly, we get

$$
\sin ^{2} \theta_{1} g\left(\widehat{\nabla}_{X} Y, V\right)=-g\left(\nabla_{X}^{\perp} N T Y, V\right)-g\left(A_{N Y} X, t V\right)+g\left(\nabla_{X}^{\perp} N Y, n V\right) .
$$

Thus, from (4.7) and (4.8), we have the assertions.

In a similar way, we obtain the following case for the distribution $D_{2} \oplus\langle\xi\rangle$ :

Theorem 4.9 Let $M$ be a proper quasi-bi-slant submanifold of a Sasakian manifold $\widehat{M}$. Then, the distribution $D_{2} \oplus\langle\xi\rangle$ defines a totally geodesic foliation on $M$ if and only if

$$
\begin{aligned}
g\left(\nabla_{X}^{\perp} N Y, N Q Z\right) & =g\left(A_{N Y} X, T P Z+T Q Z\right)-g\left(A_{N T Y} X, Z\right) \text { and } \\
g\left(A_{N Y} X, t V\right) & =g\left(\nabla_{X}^{\perp} N Y, n V\right)-g\left(\nabla_{X}^{\perp} N T Y, V\right)
\end{aligned}
$$

for any $X, Y \in \Gamma\left(D_{2} \oplus\langle\xi\rangle\right), Z \in \Gamma\left(D \oplus D_{1}\right)$ and $V \in \Gamma\left(T^{\perp} M\right)$.

\section{Quasi-bi-slant submanifolds with parallel canonical structures}

In this section, we obtain some results for the quasi-bi-slant submanifolds with parallel canonical structures. Let $M$ be a proper quasi-bi-slant submanifold of a Sasakian manifold $\widehat{M}$. Now, we define the covariant derivative of tangential and normal components of (3.2) and (3.7) as follows:

$$
\begin{aligned}
& \left(\widehat{\nabla}_{X} T\right) Y=\nabla_{X} T Y-T \nabla_{X} Y, \\
& \left(\widehat{\nabla}_{X} N\right) Y=\nabla_{X}^{\perp} N Y-N \nabla_{X} Y, \\
& \left(\widehat{\nabla}_{X} t\right) V=\nabla_{X} t V-t \nabla_{X}^{\perp} V
\end{aligned}
$$

and

$$
\left(\widehat{\nabla}_{X} n\right) V=\nabla_{X}^{\perp} n V-n \nabla_{X}^{\perp} V
$$

for any $X, Y \in \Gamma(T M)$ and $V \in \Gamma\left(T^{\perp} M\right)$.

Then, the endomorphism $T$ (resp., $N$ ) and the endomorphism $t$ (resp., $n$ ) are parallel if $\widehat{\nabla} T \equiv 0$ (resp., $\widehat{\nabla} N \equiv 0)$ and $\widehat{\nabla} t \equiv 0$ (resp., $\widehat{\nabla} n \equiv 0)$, respectively.

Making use of Lemma 3.8 and (5.1)-(5.4), we have the following lemma:

Lemma 5.1 Let $M$ be a quasi-bi-slant submanifold of a Sasakian manifold $\widehat{M}$. Then, we obtain

$$
\begin{aligned}
& \left(\widehat{\nabla}_{X} T\right) Y=A_{N Y} X+\operatorname{th}(X, Y)+g(X, Y) \xi-\eta(Y) X, \\
& \left(\widehat{\nabla}_{X} N\right) Y=n h(X, Y)-h(X, T Y), \\
& \left(\widehat{\nabla}_{X} t\right) V=A_{n V} X-T A_{V} X
\end{aligned}
$$

and

$$
\left(\widehat{\nabla}_{X} n\right) V=-h(X, t V)-N A_{V} X
$$

for any $X, Y \in \Gamma(T M)$ and $V \in \Gamma\left(T^{\perp} M\right)$.

Proposition 5.2 Let $M$ be a quasi-bi-slant submanifold of a Sasakian manifold $\widehat{M}$. Then, $T$ is parallel if and only if the shape operator satisfies

$$
A_{N Y} Z-A_{N Z} Y=\eta(Y) Z-\eta(Z) Y
$$

for any $Y, Z \in \Gamma(T M)$. 
Proof For any $X, Y, Z \in \Gamma(T M)$, from (5.5), we have

$$
\begin{aligned}
g\left(\left(\widehat{\nabla}_{X} T\right) Y, Z\right) & =g\left(A_{N Y} X+t h(X, Y)+g(X, Y) \xi-\eta(Y) X, Z\right) \\
& =g\left(A_{N Y} X, Z\right)-g(h(X, Y), \phi Z)+g(X, Y) \eta(Z)-g(X, Z) \eta(Y) \\
& =g(h(X, Z), N Y)-g(h(X, Y), \phi Z)+g(X, Y) \eta(Z)-g(X, Z) \eta(Y) \\
& =g(h(X, Z), N Y)-g(h(X, Y), N Z)+g(X, Y) \eta(Z)-g(X, Z) \eta(Y) \\
& =g\left(A_{N Y} Z-A_{N Z} Y+\eta(Z) Y-\eta(Y) Z, X\right) .
\end{aligned}
$$

From this, we obtain the proposition.

From (5.6), we have the following proposition:

Proposition 5.3 Let $M$ be a quasi-bi-slant submanifold of a Sasakian manifold $\widehat{M}$. Then, $N$ is parallel if and only if

$$
n h(X, Y)=h(X, T Y)
$$

or equivalently

$$
A_{n V} Y=-A_{V} T Y
$$

for any $X, Y, \in \Gamma(T M)$ and $V \in \Gamma\left(T^{\perp} M\right)$.

Theorem 5.4 Let $M$ be a quasi-bi-slant submanifold of a Sasakian manifold $\widehat{M}$. Then, $N$ is parallel if and only if $t$ is parallel.

Proof For any $X, Y \in \Gamma(T M)$ and $V \in \Gamma\left(T^{\perp} M\right)$, using (2.5), (2.6), and (5.6), we have

$$
\begin{aligned}
g\left(\left(\widehat{\nabla}_{X} N\right) Y, V\right) & =g(n h(X, Y)-h(X, T Y), V) \\
& =-g(h(X, Y), \phi V)-g(h(X, T Y), V) \\
& =-g(h(X, Y), n V)-g\left(\widehat{\nabla}_{X} T Y, V\right) \\
& =-g\left(A_{n V} X, Y\right)+g\left(T Y, \widehat{\nabla}_{X} V\right) \\
& =-g\left(A_{n V} X, Y\right)-g\left(T Y, A_{V} X\right) \\
& =-g\left(A_{n V} X, Y\right)+g\left(T A_{V} X, Y\right) \\
& =-g\left(\left(\widehat{\nabla}_{X} t\right) V, Y\right) .
\end{aligned}
$$

which gives the assertion.

\section{Example}

Now, we give a non-trivial example of proper quasi-bi-slant submanifold in $\mathbb{R}^{11}$.

Consider the Euclidean space $\mathbb{R}^{11}$ with coordinates $\left(x_{1}, \ldots, x_{5}, y_{1} \ldots, y_{5}, z\right)$. Define an almost contact structure on $\mathbb{R}^{11}$ as follows:

$$
\begin{aligned}
& \phi\left(\sum_{i=1}^{5}\left(X^{i} \frac{\partial}{\partial x_{i}}+Y^{i} \frac{\partial}{\partial y_{i}}\right)+Z \frac{\partial}{\partial z}\right)=\sum_{i=1}^{5} Y^{i} \frac{\partial}{\partial x_{i}}-\sum_{i=1}^{5} X^{i} \frac{\partial}{\partial y_{i}}+\sum_{i=1}^{5} Y^{i} y_{i} \frac{\partial}{\partial z} \\
& g=(\eta \otimes \eta)+\frac{1}{4} \sum_{i=1}^{5}\left(d x_{i} \otimes d x_{i}+d y_{i} \otimes d y_{i}\right), \\
& \eta=\frac{1}{2}\left(d z-\sum_{i=1}^{5} y_{i} d x_{i}\right), \quad \xi=2 \frac{\partial}{\partial z} .
\end{aligned}
$$

Then, $\left(\mathbb{R}^{11}, \phi, \xi, \eta, g\right)$ becomes a Sasakian manifold of dimension 11 . We choose the vector fields

$$
e_{i}=2 \frac{\partial}{\partial y_{i}}, e_{5+i}=2\left(\frac{\partial}{\partial x_{i}}+y_{i} \frac{\partial}{\partial z}\right), e_{11}=\xi=2 \frac{\partial}{\partial z}, \quad \text { for } i=1,2, \ldots, 5 .
$$


Then, $\left\{e_{1}, e_{2}, \ldots, e_{11}\right\}$ forms an orthonormal basis at each point of tangent space of $\mathbb{R}^{11}$.

Now, we consider a submanifold $M$ of $\mathbb{R}^{11}$ defined by immersion $\psi$ as follows:

$$
\psi(u, v, w, r, s, t, q)=\left(u, \frac{w}{2}, 0, \frac{s}{2}, 0, v, \frac{\sqrt{3}}{2} r, \frac{r}{2}, \frac{t}{2}, \frac{t}{2}, q\right) .
$$

If we take

$$
\begin{aligned}
& X_{1}=e_{1}, \quad X_{2}=e_{6}, \quad X_{3}=\frac{1}{2} e_{2}, \\
& X_{4}=\frac{1}{2}\left(\sqrt{3} e_{7}+e_{8}\right), \quad X_{5}=\frac{1}{2} e_{4}, \\
& X_{6}=\frac{1}{2}\left(e_{9}+e_{10}\right), \quad X_{7}=e_{11} .
\end{aligned}
$$

Then, the set $\left\{X_{1}, X_{2}, X_{3}, X_{4}, X_{5}, X_{6}, X_{7}\right\}$ restricted over $M$ spans the tangent bundle $T M$ of $M$.

Obviously, we get

$$
\begin{aligned}
& \phi X_{1}=e_{6}, \quad \phi X_{2}=-e_{1}, \quad \phi X_{3}=\frac{1}{2} e_{7}, \\
& \phi X_{4}=-\frac{1}{2}\left(\sqrt{3} e_{2}+e_{3}\right), \quad \phi X_{5}=\frac{1}{2} e_{9}, \\
& \phi X_{6}=-\frac{1}{2}\left(e_{4}+e_{5}\right), \quad \phi X_{7}=0 .
\end{aligned}
$$

Now, let us put $D=\operatorname{Span}\left\{X_{1}, X_{2}\right\}, D_{1}=\operatorname{Span}\left\{X_{3}, X_{4}\right\}, D_{2}=\operatorname{Span}\left\{X_{5}, X_{6}\right\}$.

Now, for any $X \in T M$, we have

$$
\begin{aligned}
X & =a_{1} X_{1}+a_{2} X_{2}+a_{3} X_{3}+a_{4} X_{4}+a_{5} X_{5}+a_{6} X_{6}+a_{7} X_{7} \\
& =a_{1} e_{1}+a_{2} e_{6}+\frac{a_{3}}{2} e_{2}+\frac{a_{4}}{2}\left(\sqrt{3} e_{7}+e_{8}\right)+\frac{a_{5}}{2} e_{4}+\frac{a_{6}}{2}\left(e_{9}+e_{10}\right)+a_{7} e_{11},
\end{aligned}
$$

where $a_{1}, a_{2} \ldots, a_{7}$ are $C^{\infty}$-functions on $M$.

Therefore, $P X=a_{1} e_{1}+a_{2} e_{6}, Q X=\frac{a_{3}}{2} e_{2}+\frac{a_{4}}{2}\left(\sqrt{3} e_{7}+e_{8}\right)$, and $R X=\frac{a_{5}}{2} e_{4}+\frac{a_{6}}{2}\left(e_{9}+e_{10}\right)$.

By the use of (6.1), we have

$$
\begin{aligned}
\phi X= & a_{1} e_{6}-a_{2} e_{1}+\frac{a_{3}}{2} e_{7}-\frac{a_{4}}{2}\left(\sqrt{3} e_{2}+e_{3}\right)+\frac{a_{5}}{2} e_{9}-\frac{a_{6}}{2}\left(e_{4}+e_{5}\right) \\
= & a_{1} e_{6}-a_{2} e_{1}+\frac{a_{3}}{4 \sqrt{3}}\left(\sqrt{3} e_{7}+e_{8}\right)+\frac{a_{3}}{4 \sqrt{3}}\left(\sqrt{3} e_{7}-e_{8}\right)-\frac{a_{4}}{2}\left(\sqrt{3} e_{2}+e_{3}\right) \\
& +\frac{a_{5}}{4}\left(e_{9}+e_{10}\right)+\frac{a_{5}}{4}\left(e_{9}-e_{10}\right)-\frac{a_{6}}{2}\left(e_{4}+e_{5}\right) .
\end{aligned}
$$

Therefore, we have

$$
\begin{aligned}
& T X=a_{1} e_{6}-a_{2} e_{1}+\frac{a_{3}}{4 \sqrt{3}}\left(\sqrt{3} e_{7}+e_{8}\right)-\frac{\sqrt{3} a_{4}}{2} e_{2}+\frac{a_{5}}{4}\left(e_{9}+e_{10}\right)-\frac{a_{6}}{2} e_{4}, \\
& N X=\frac{a_{3}}{4 \sqrt{3}}\left(\sqrt{3} e_{7}-e_{8}\right)-\frac{a_{4}}{2} e_{3}+\frac{a_{5}}{4}\left(e_{9}-e_{10}\right)-\frac{a_{6}}{2} e_{5} .
\end{aligned}
$$

Now, using above relations, we have

$$
\begin{aligned}
& T P X=a_{1} e_{6}-a_{2} e_{1}, \quad T Q X=\frac{a_{3}}{4 \sqrt{3}}\left(\sqrt{3} e_{7}+e_{8}\right)-\frac{\sqrt{3} a_{4}}{2} e_{2}, \quad T R X=\frac{a_{5}}{4}\left(e_{9}+e_{10}\right)-\frac{a_{6}}{2} e_{4}, \\
& N P X=0, \quad N Q X=\frac{a_{3}}{4 \sqrt{3}}\left(\sqrt{3} e_{7}-e_{8}\right)-\frac{a_{4}}{2} e_{3}, \quad N R X=\frac{a_{5}}{4}\left(e_{9}-e_{10}\right)-\frac{a_{6}}{2} e_{5} .
\end{aligned}
$$


If we take $Z_{1}=\frac{1}{2}\left(\sqrt{3} e_{7}-e_{8}\right), Z_{2}=\frac{1}{2} e_{3}, Z_{3}=\frac{1}{2}\left(e_{9}-e_{10}\right)$, and $Z_{4}=\frac{1}{2} e_{5}$. Then

$$
T^{\perp} M=\left\langle Z_{1}, Z_{2}\right\rangle \oplus\left\langle Z_{3}, Z_{4}\right\rangle .
$$

Here, in this example, $\mu=\{0\}$.

Now, for any $V \in T^{\perp} M$, we have $V=b_{1} Z_{1}+b_{2} Z_{2}+b_{3} Z_{3}+b_{4} Z_{4}$, where $b_{1}, b_{2}, b_{3}, b_{4}$ are $C^{\infty}$-functions on $M$. Therefore

$$
V=\frac{b_{1}}{2}\left(\sqrt{3} e_{7}-e_{8}\right)+\frac{b_{2}}{2} e_{3}+\frac{b_{3}}{2}\left(e_{9}-e_{10}\right)+\frac{b_{4}}{2} e_{5}
$$

Using (6.1), we get

$$
\begin{aligned}
\phi V= & -\frac{b_{1}}{2}\left(\sqrt{3} e_{2}-e_{3}\right)+\frac{b_{2}}{2} e_{8}-\frac{b_{3}}{2}\left(e_{4}-e_{5}\right)+\frac{b_{4}}{2} e_{10} \\
= & -\frac{b_{1}}{2}\left(\sqrt{3} e_{2}-e_{3}\right)+\frac{b_{2}}{4}\left(\sqrt{3} e_{7}+e_{8}\right)-\frac{b_{2}}{4}\left(\sqrt{3} e_{7}-e_{8}\right) \\
& -\frac{b_{3}}{2}\left(e_{4}-e_{5}\right)+\frac{b_{4}}{4}\left(e_{9}+e_{10}\right)-\frac{b_{4}}{4}\left(e_{9}-e_{10}\right) .
\end{aligned}
$$

Therefore, we have

$$
\begin{aligned}
& t V=-\frac{b_{1}}{2} \sqrt{3} e_{2}+\frac{b_{2}}{4}\left(\sqrt{3} e_{7}+e_{8}\right)-\frac{b_{3}}{2} e_{4}+\frac{b_{4}}{4}\left(e_{9}+e_{10}\right), \\
& n V=\frac{b_{1}}{2} e_{3}-\frac{b_{2}}{4}\left(\sqrt{3} e_{7}-e_{8}\right)+\frac{b_{3}}{2} e_{5}-\frac{b_{4}}{4}\left(e_{9}-e_{10}\right) .
\end{aligned}
$$

Using the property of Lie bracket, we have

$$
\begin{aligned}
{\left[e_{i}, e_{5+i}\right] } & =e_{11} \quad \text { for } i=1,2, \ldots, 5, \\
\text { otherwise }\left[e_{i}, e_{j}\right] & =0 .
\end{aligned}
$$

Let $\widehat{\nabla}$ be the Levi-Civita connection of Riemannian metric $g$ which is given by the Koszul's formula defined by

$$
2 g\left(\widehat{\nabla}_{X} Y, Z\right)=X g(Y, Z)+Y g(Z, X)-Z g(X, Y)-g(X,[Y, Z])-g(Y,[X, Z])+g(Z,[X, Y])
$$

Using Koszul's formula, we can easily obtain non-zero components of Levi-Civita connection $\widehat{\nabla}$ as follows:

$$
\begin{aligned}
\widehat{\nabla}_{e_{i}} e_{11} & =-\frac{1}{2} e_{5+i}, \quad \widehat{\nabla}_{e_{5+i}} e_{11}=\frac{1}{2} e_{i}, \\
\widehat{\nabla}_{e_{i}} e_{5+i} & =\frac{1}{2} e_{11}, \quad \widehat{\nabla}_{e_{5+i}} e_{i}=-\frac{1}{2} e_{11}, \\
\widehat{\nabla}_{e_{11}} e_{i} & =-\frac{1}{2} e_{5+i}, \quad \widehat{\nabla}_{e_{11}} e_{5+i}=\frac{1}{2} e_{i},
\end{aligned}
$$

for $i=1,2, \ldots, 5$.

By the use of (2.5) and (6.5), we can easily obtain non-zero components of second fundamental form $h$ as follows:

$$
\begin{aligned}
h\left(e_{2}, e_{11}\right) & =h\left(e_{11}, e_{2}\right)=-\frac{1}{2 \sqrt{3}} Z_{1} \\
h\left(e_{4}, e_{11}\right) & =h\left(e_{11}, e_{4}\right)=-\frac{1}{2} Z_{3} \\
h\left(e_{8}, e_{11}\right) & =h\left(e_{11}, e_{8}\right)=Z_{2} \\
h\left(e_{10}, e_{11}\right) & =h\left(e_{11}, e_{10}\right)=Z_{4} .
\end{aligned}
$$


Now, for any $X, Y \in T M$, we have

$$
\begin{aligned}
X & =a_{1} X_{1}+a_{2} X_{2}+a_{3} X_{3}+a_{4} X_{4}+a_{5} X_{5}+a_{6} X_{6}+a_{7} X_{7} \\
& =a_{1} e_{1}+a_{2} e_{6}+\frac{a_{3}}{2} e_{2}+\frac{a_{4}}{2}\left(\sqrt{3} e_{7}+e_{8}\right)+\frac{a_{5}}{2} e_{4}+\frac{a_{6}}{2}\left(e_{9}+e_{10}\right)+a_{7} e_{11}, \\
Y & =c_{1} e_{1}+c_{2} e_{6}+\frac{c_{3}}{2} e_{2}+\frac{c_{4}}{2}\left(\sqrt{3} e_{7}+e_{8}\right)+\frac{c_{5}}{2} e_{4}+\frac{c_{6}}{2}\left(e_{9}+e_{10}\right)+c_{7} e_{11},
\end{aligned}
$$

where $a_{1}, a_{2} \ldots, a_{7}, c_{1}, \ldots, c_{7}$ are $C^{\infty}$-functions on $M$.

Therefore, by the use of (6.6) and (6.7), we have

$$
\begin{aligned}
h(X, Y)= & -\frac{1}{4 \sqrt{3}}\left(a_{3} c_{7}+a_{7} c_{3}\right) Z_{1}+\frac{1}{2}\left(a_{4} c_{7}+a_{7} c_{4}\right) Z_{2} \\
& -\frac{1}{4}\left(a_{5} c_{7}+a_{7} c_{5}\right) Z_{3}+\frac{1}{2}\left(a_{6} c_{7}+a_{7} c_{6}\right) Z_{4} .
\end{aligned}
$$

Using (2.7), (6.2), and (6.8), we can find shape operator $A$ as follows:

$$
\begin{aligned}
A_{V} X= & -\frac{1}{\sqrt{3}} a_{7} b_{1} X_{3}+\frac{1}{8} a_{7} b_{2} X_{4}-\frac{1}{2} a_{7} b_{3} X_{5}+\frac{1}{4} a_{7} b_{4} X_{6} \\
& -\left(\frac{1}{4 \sqrt{3}} a_{3} b_{1}-\frac{1}{8} a_{4} b_{2}+\frac{1}{8} a_{5} b_{3}-\frac{1}{8} a_{6} b_{4}\right) X_{7} .
\end{aligned}
$$

Also one can easily check that the distribution $D$ is invariant, the distribution $D_{1}$ is slant with slant angle $\frac{\pi}{6}$, and the distribution $D_{2}$ is also slant with slant angle $\frac{\pi}{4}$. Also, the distributions $D, D_{1}$ and $D_{2}$ satisfy the definition of quasi-bi-slant submanifold of Sasakian manifold. Hence, the submanifold $M$ defined by $\psi$ is a proper quasi-bi-slant submanifold of $\mathbb{R}^{11}$ with $\frac{\pi}{6}, \frac{\pi}{4}$ as its bi-slant angles.

Clearly, the distributions $D, D_{1}$, and $D_{2}$ are not integrable. Hence, the given example verifies Proposition 4.1.

Open Access This article is licensed under a Creative Commons Attribution 4.0 International License, which permits use, sharing, adaptation, distribution and reproduction in any medium or format, as long as you give appropriate credit to the original author(s) and the source, provide a link to the Creative Commons licence, and indicate if changes were made. The images or other third party material in this article are included in the article's Creative Commons licence, unless indicated otherwise in a credit line to the material. If material is not included in the article's Creative Commons licence and your intended use is not permitted by statutory regulation or exceeds the permitted use, you will need to obtain permission directly from the copyright holder. To view a copy of this licence, visit http://creativecommons.org/licenses/by/4.0/.

\section{References}

1. Benjancu, A.; Papaghiuc, N.: Semi-invariant submanifolds of a Sasakian manifold. An. St. Univ. AI. I. Cuza. Iasi. Math. (N.S.) 27, 163-170 (1981)

2. Blair, D.E.: Contact Manifold in Riemannian Geometry, vol. 509. Lecture Notes in MathematicsSpringer, New York (1976)

3. Cabrerizo, J.L.; Carriazo, A.; Fernandez, L.M.; Fernandez, M.: Semi-slant submanifolds of a Sasakian manifold. Geom. Dedicata 78, 183-199 (1999)

4. Cabrerizo, J.L.; Carriazo, A.; Fernandez, L.M.; Fernandez, M.: Slant submanifolds in Sasakian manifolds. Glasg. Math. J. 42, 125-138 (2000)

5. Chen, B.Y.: Geometry of Slant Submanifolds. Katholieke Universiteit, Leuven (1990)

6. Chen, B.Y.: Differential geometry of real submanifold in Kaehler manifold. Monatsh. Math. 91, 257-274 (1981)

7. Chen, B.Y.: Slant immersions. Bull. Aust. Math. Soc. 41(1), 135-147 (1990)

8. Chen, B.Y.; Tazawa, Y.: Slant surfaces with codimension 2. Ann. Fac. Sci. Toulouse Math. X I(3), 29-43 (1990)

9. Chen, B.Y.; Tazawa, Y.: Slant submanifolds in complex Euclidean spaces. Tokyo J. Math. 14, 101-120 (1991)

10. De, U.C.; Shaikh, A.A.: Complex Manifolds and Contact Manifolds. Narosa Publishing House, New Delhi (2009)

11. Gupta, R.S.; Haider, S.M.K.; Shahid, M.H.: Slant submanifolds of cosymplectic manifold. An. Stiint. Univ. Al. I. Cuza Iasi. Mat. (N.S.) 50(1), 33-50 (2004)

12. Khan, V.A.; Khan, M.A.: Pseudo-slant submanifolds of Sasakian manifold. Indian J. Pure Appl. Math. 38(1), 31-42 (2007)

13. Kon, M.: Remarks on anti-invariant submanifolds of a Sasakian manifold. Tensor (N.S.) 30, 239-245 (1976)

14. Lone, M.A.; Lone, M.S.; Sahid, M.H.: Hemi-slant submanifolds of cosymplectic manifolds. Cogent Math. 3, 120-143 (2016)

15. Lotta, A.: Slant submanifold in contact geometry. Bull. Math. Soc. Rom. 39, 183-198 (1996)

16. Lotta, A.: Three-dimensional slant submanifolds of K-contact manifolds. Balk. J. Geom. Appl. 3(1), 37-51 (1998) 
17. Park, K.S.; Prasad, R.: Semi-slant submersions. Bull. Korean Math. Soc. 50(3), 951-962 (2013)

18. Prasad, R.; Verma, S.K.; Kumar, S.: Quasi hemi-slant submanifolds of Sasakian manifolds. J. Math. Comput. Sci. 10(2), 418-435 (2020)

19. Prasad, R.; Verma, S.K.; Kumar, S.; Chaubey, S.K.: Quasi hemi-slant submanifolds of cosymplectic manifolds. Korean J. Math. 28(2), 257-273 (2020)

20. Sahin, B.: Warped product submanifolds of a Kaehler manifold with a slant factor. Ann. Pol. Math. 95, 107-126 (2009)

21. Sahin, F.: Cohomology of hemi-slant submanifolds of a Kaehler manifold. J. Adv. Stud. Topol. 5, 27-31 (2014)

22. Uddin, S.; Chen, B.Y.; Al-Solamy, F.R.: Warped product bi-slant immersions in Kaehler manifolds. Mediterr. J. Math. 14(2), 14-95 (2017)

Publisher's Note Springer Nature remains neutral with regard to jurisdictional claims in published maps and institutional affiliations. 